\title{
Some New Generalized Retarded Gronwall-Like Inequalities and Their Applications in Nonlinear Systems
}

\author{
Haiyong Qin, ${ }^{1}$ Xin Zuo, ${ }^{2}$ and Jianwei Liu ${ }^{2}$ \\ ${ }^{1}$ School of Mathematics, Qilu Normal University, Jinan, Shandong 250013, China \\ ${ }^{2}$ Department of Automation, China University of Petroleum (Beijing), Changping, Beijing 102249, China \\ Correspondence should be addressed to Haiyong Qin; qhymath@hotmail.com
}

Received 15 November 2015; Accepted 15 February 2016

Academic Editor: Petko Petkov

Copyright (c) 2016 Haiyong Qin et al. This is an open access article distributed under the Creative Commons Attribution License, which permits unrestricted use, distribution, and reproduction in any medium, provided the original work is properly cited.

\begin{abstract}
The Gronwall inequalities are of significance in mathematics and engineering. This paper generalizes the Gronwall-like inequalities from different perspectives. Using the proposed inequalities, the difficulties to discuss the controllability of integrodifferential systems of mixed type can be solved. Meanwhile, two examples as their applications are also given to show the effectiveness of our main results.
\end{abstract}

\section{Introduction}

Integral inequalities provide a powerful and important tool in the study of qualitative properties of solutions of nonlinear differential, integral, and integrodifferential equations, as well as in the modeling of science and engineering problems (see [1]). One of the most famous inequalities of this type is known as "Gronwall's inequality," "Bellman's inequality," or "Gronwall-Bellman's inequality" (see [2,3]). Recently, the celebrated Gronwall inequality and its generalizations play increasingly important roles in the qualitative analysis of differential, integral, and integrodifferential equations. Based on the different purposes, many researchers put their efforts in exploring new inequalities and their applications in many fields, and many useful Gronwall-like integral inequalities have been established in various problems (see [4-17]).

Lipovan [18] proved a Gronwall-like inequality, and in order to show its applications, Lipovan applied his main results to the qualitative analysis of solutions to certain integral equations, functional differential equations, and retarded differential equations. Ye et al. [19] gave a generalized Gronwall inequality with singularity which can be applied to weakly singular Volterra integral equations and fractional integral and integrodifferential equations. Liu [20] proved a comparison result, which is widely known later and always used to provide explicit bounds on solutions and estimate on noncompactness. In addition, some existence theorems of solutions and iterative approximation of the unique solution for the nonlinear integrodifferential equations of mixed type are obtained. However, it is worth mentioning that it is difficult to deal with integrodifferential systems which include a Fredholm operator in nonlinearity unless powerful integral inequalities are established.

In this paper, we prove a generalization of the Gronwall inequality. As an application, we show that the inequality can be applied to the controllability analysis of abstract control system and existence analysis. Sufficient conditions ensuring the controllability of certain impulsive integrodifferential system of mixed type are obtained. The main difficulties from the Fredholm operator can be overcome.

The rest of the paper is organized as follows. In Section 2, we present some preliminaries and lemmas and prove some generalized Gronwall-like inequalities. In Section 3, we discuss the controllability of impulsive integrodifferential systems of mixed type in a Banach space as an application. In Section 4, we give another example to illustrate the application of our main results vividly. Finally, conclusions are given in Section 5.

\section{Integral Inequalities}

In this section, we present a generalization of the Gronwalllike inequality which can be called a comparison result 
in many literatures (see [20]). Unless otherwise stated, we denote $\mathbb{R}_{+}=(0,+\infty), \mathbb{R}^{+}=[0,+\infty)$ in this paper.

Lemma 1. Suppose that $u, f, g \in C\left(\left[t_{0}, T\right), \mathbb{R}^{+}\right)$. Let $w \in$ $C\left(\mathbb{R}^{+}, \mathbb{R}^{+}\right)$be nondecreasing with $w(u)>0$ for $u>0$ and let $\alpha, \beta \in C^{1}\left(\left[t_{0}, T\right),\left[t_{0}, T\right)\right)$ be nondecreasing with $\alpha(t), \beta(t) \leq t$ on $\left[t_{0}, T\right)$. If

$$
\begin{aligned}
u(t) \leq & m_{1}(t)+m_{2}(t) \int_{\alpha\left(t_{0}\right)}^{\alpha(t)} f(s) w(u(s)) d s \\
& +m_{3}(t) \int_{\beta\left(t_{0}\right)}^{\beta(t)} g(s) w(u(s)) d s,
\end{aligned}
$$

where $m_{i}(t)$ is a nonnegative, continuous function defined on $t_{0} \leq t<T$, and there exist nonnegative constants $M_{i}$ such that $m_{i}(t) \leq M_{i}(i=1,2,3)$, then, for $t_{0} \leq t<t_{1}$, one has

$$
\begin{aligned}
u(t) & \leq G^{-}\left(G\left(M_{1}\right)+M_{2} \int_{\alpha\left(t_{0}\right)}^{\alpha(t)} f(s) d s\right. \\
& \left.+M_{3} \int_{\beta\left(t_{0}\right)}^{\beta(t)} g(s) d s\right),
\end{aligned}
$$

where $G(r)=\int_{1}^{r}(1 / w(s)) d s, r>0, t_{1} \in\left(t_{0}, T\right)$ is chosen so that

$$
\begin{aligned}
& G\left(M_{1}\right)+M_{2} \int_{\alpha\left(t_{0}\right)}^{\alpha(t)} f(s) d s+M_{3} \int_{\beta\left(t_{0}\right)}^{\beta(t)} g(s) d s \\
& \quad \in \operatorname{Dom}\left(G^{-}\right)
\end{aligned}
$$

for all $t$ lying in the interval $\left[t_{0}, t_{1}\right)$.

Proof. Noting the conditions we imposed, we have

$$
\begin{gathered}
u(t) \leq M_{1}+M_{2} \int_{\alpha\left(t_{0}\right)}^{\alpha(t)} f(s) w(u(s)) d s \\
+M_{3} \int_{\beta\left(t_{0}\right)}^{\beta(t)} g(s) w(u(s)) d s .
\end{gathered}
$$

Let us denote

$$
\begin{aligned}
U(t)= & M_{1}+M_{2} \int_{\alpha\left(t_{0}\right)}^{\alpha(t)} f(s) w(u(s)) d s \\
& +M_{3} \int_{\beta\left(t_{0}\right)}^{\beta(t)} g(s) w(u(s)) d s .
\end{aligned}
$$

Obviously, we have $U\left(t_{0}\right)=M_{1}$ and

$$
\begin{aligned}
U^{\prime}(t)= & M_{2} f(\alpha(t)) w(u(\alpha(t))) \alpha^{\prime}(t) \\
& +M_{3} g(\beta(t)) w(u(\beta(t))) \beta^{\prime}(t) .
\end{aligned}
$$

Since $\alpha(t) \leq t$ and $\beta(t) \leq t$ on $\left[t_{0}, T\right)$, then

$$
\begin{aligned}
U^{\prime}(t) \leq & M_{2} f(\alpha(t)) w(U(t)) \alpha^{\prime}(t) \\
& +M_{3} g(\beta(t)) w(U(t)) \beta^{\prime}(t) .
\end{aligned}
$$

By the definitions of $G$, we obtain that

$$
\frac{d}{d t} G(U(t)) \leq M_{2} f(\alpha(t)) \alpha^{\prime}(t)+M_{3} g(\beta(t)) \beta^{\prime}(t)
$$

integrate both sides, and we conclude that

$$
\begin{aligned}
G(U(t)) \leq & G\left(M_{1}\right)+M_{2} \int_{\alpha\left(t_{0}\right)}^{\alpha(t)} f(s) d s \\
& +M_{3} \int_{\beta\left(t_{0}\right)}^{\beta(t)} g(s) d s .
\end{aligned}
$$

Because $G^{-}$is increasing on $\operatorname{Dom}\left(G^{-}\right)$, we get

$$
\begin{aligned}
& u(t) \leq G^{-}\left(G\left(M_{1}\right)+M_{2} \int_{\alpha\left(t_{0}\right)}^{\alpha(t)} f(s) d s\right. \\
& \left.+M_{3} \int_{\beta\left(t_{0}\right)}^{\beta(t)} g(s) d s\right) .
\end{aligned}
$$

Remark 2. Next, we shall show that Lemma 1 generalizes some existing results:

(1) For $m_{1}(t) \equiv k, m_{2}(t) \equiv 1$, and $m_{3}(t) \equiv 0$, we obtain theorem in [18]. Further supposing that $\alpha(t) \equiv t$, we get the celebrated Bihari's inequality.

(2) Set $w(u) \equiv u$. Note that $G(u)=\int_{1}^{\infty}(1 / s) d s=\infty$; then the previous result (2) holds.

(3) Compared with Theorem 1 in [19], this lemma has a different range of applications.

Corollary 3. Suppose that $u, f, g \in C\left(\left[t_{0}, T\right), \mathbb{R}^{+}\right)$. Let $\alpha$, $\beta \in C^{1}\left(\left[t_{0}, T\right),\left[t_{0}, T\right)\right)$ be nondecreasing with $\alpha(t), \beta(t) \leq t$ on $\left[t_{0}, T\right)$. If

$$
u(t) \leq M_{1}+M_{2} \int_{\alpha\left(t_{0}\right)}^{\alpha(t)} f(s) u(s) d s
$$

$$
+M_{3} \int_{\beta\left(t_{0}\right)}^{\beta(t)} g(s) u(s) d s
$$

where $M_{i}(i=1,2,3)$ is nonnegative constants, then, for $t_{0} \leq$ $t<T$, one has

$u(t)$

$$
\leq M_{1} \exp \left(M_{2} \int_{\alpha\left(t_{0}\right)}^{\alpha(t)} f(s) d s+M_{3} \int_{\beta\left(t_{0}\right)}^{\beta(t)} g(s) d s\right) .
$$

Remark 4. It is easy to get the following results.

(1) Assume that $M_{2}=1$ and $M_{3}=0$; we know that corollary in [18] is valid.

(2) With $M_{3}=0$ and $\alpha(t)=t$, we obtain the celebrated Gronwall-Bellman inequality. 
Theorem 5. Suppose that $u, f, g \in C\left(\left[t_{0}, T\right), \mathbb{R}^{+}\right)$. Let $w \in$ $C\left(\mathbb{R}^{+}, \mathbb{R}^{+}\right)$be nondecreasing with $w(u)>0$ for $u>0$ and let $\alpha, \beta \in C^{1}\left(\left[t_{0}, T\right),\left[t_{0}, T\right)\right)$ be nondecreasing with $\alpha(t), \beta(t) \leq t$ on $\left[t_{0}, T\right)$. If

$$
\begin{aligned}
u(t) \leq & M_{1}+M_{2} \int_{\alpha\left(t_{0}\right)}^{\alpha(t)} f(s) w(u(s)) d s \\
& +M_{3} \int_{\beta\left(t_{0}\right)}^{\beta(t)} g(s) w(u(s)) d s \\
& +M_{4} \int_{t_{0}}^{T}[u(s)]^{\lambda} d s,
\end{aligned}
$$

where $M_{i}(i=1,2,3,4)$ are nonnegative constants, $0 \leq \lambda<1$, and $\int_{1}^{\infty}(1 / w(s)) d s=\infty$, then, for $t_{0} \leq t<T$, one has

$$
u(t) \leq m^{-}(0)
$$

where $m(s)=\left(2 s-M M_{1}\right)^{1-\lambda}-s^{1-\lambda}-(1-\lambda) M M_{4} T, M$ is a positive constant, and $m^{-}(\cdot)$ represents the inverse of $m(\cdot)$.

Proof. By Lemma 1 and Remark 2 (2), for $t_{0} \leq t<T$, there exists a constant $M>0$ such that

$$
u(t) \leq M\left(M_{1}+M_{4} \int_{t_{0}}^{T}[u(s)]^{\lambda} d s\right)
$$

Define

$$
\begin{aligned}
& p(t) \\
& =M\left(M_{1}+M_{4} \int_{t_{0}}^{t}[u(s)]^{\lambda} d s+M_{4} \int_{t_{0}}^{T}[u(s)]^{\lambda} d s\right) ;
\end{aligned}
$$

we have

$$
\begin{aligned}
& p\left(t_{0}\right)=M M_{1}+M M_{4} \int_{t_{0}}^{T}[u(s)]^{\lambda} d s, \\
& p^{\prime}(t) \leq M M_{4}[p(t)]^{\lambda} .
\end{aligned}
$$

Integrating from $t_{0}$ to $t$, we get

$$
[p(t)]^{1-\lambda}-\left[p\left(t_{0}\right)\right]^{1-\lambda} \leq(1-\lambda) M M_{4}\left(t-t_{0}\right) ;
$$

then

$$
p(t) \leq\left\{\left[p\left(t_{0}\right)\right]^{1-\lambda}+(1-\lambda) M M_{4}\left(t-t_{0}\right)\right\}^{1 /(1-\lambda)} .
$$

Since

$$
\begin{aligned}
& 2 p\left(t_{0}\right)-M M_{1}=p(T) \\
& \quad \leq\left\{\left[p\left(t_{0}\right)\right]^{1-\lambda}+(1-\lambda) M M_{4}\left(T-t_{0}\right)\right\}^{1 /(1-\lambda)},
\end{aligned}
$$

we can deduce that

$$
\left(2 p\left(t_{0}\right)-M M_{1}\right)^{1-\lambda}-\left[p\left(t_{0}\right)\right]^{1-\lambda} \leq(1-\lambda) M M_{4} T .
$$

Let

$$
m(s)=\left(2 s-M M_{1}\right)^{1-\lambda}-s^{1-\lambda}-(1-\lambda) M M_{4} T .
$$

Observe that $m \in C\left(\left[M M_{1} / 2, \infty\right], \mathbb{R}^{+}\right)$and

$$
\begin{aligned}
m\left(\frac{M M_{1}}{2}\right) & =-\left(\frac{M M_{1}}{2}\right)^{1-\lambda}-(1-\lambda) M M_{4} T<0, \\
\lim _{s \rightarrow 0} \frac{m(s)}{s^{1-\lambda}} & =2^{1-\lambda}-1>0 .
\end{aligned}
$$

It is easy to get that there exists a $s_{0}$ such that $m\left(s_{0}\right)=0$; then $p\left(t_{0}\right) \leq s_{0}$. Therefore

$$
u(t) \leq p\left(t_{0}\right) \leq m^{-}(0), \quad t \in\left[t_{0}, T\right) .
$$

The proof is completed.

Remark 6. (i) If $M_{1}=M_{4}=0$, we have $m^{-}\left(t_{0}\right)=0$; that is, $u(t) \equiv 0$.

(ii) Generally speaking, the spectral radius of Fredholm operators should not be less than one. However, there is no doubt that here the above inequality is satisfied as a particular case.

\section{Controllability of Differential Systems of Mixed Type}

In this section, we shall give an application to show that the proposed inequalities are useful in investigating the existence of mild solutions and controllability of differential systems of mixed type. Unfortunately, since the spectral radius of Fredholm operators should not be less than one, the inequality used in previous paper may be not suitable (see [21-26]). Therefore, more powerful integral inequalities should be established to solve the problem. In order to illustrate this problem, we consider the following impulsive integrodifferential system in a Banach space:

$$
\begin{aligned}
& x^{\prime}(t)=A(t) x(t)+f(t, x(t),(S x)(t),(T x)(t)) \\
& +(B u)(t), \quad t \in I=[0, b], \\
& \Delta x\left(t_{i}\right)=I_{i}\left(x\left(t_{i}\right)\right)=x\left(t_{i}^{+}\right)-x\left(t_{i}^{-}\right), \\
& i=1,2, \ldots, s, \\
& x(0)=x_{0},
\end{aligned}
$$

where operators $S$ and $T$ are defined as follows:

$$
\begin{aligned}
& (S x)(t)=\int_{0}^{t} k(t, s, x(s)) d s, \\
& (T x)(t)=\int_{0}^{b} h(t, s, x(s)) d s .
\end{aligned}
$$

$A(t)$ is a family of linear operators which generates an evolution operator

$$
\begin{aligned}
G: \Delta & =\{(t, s) \in[0, b] \times[0, b]: 0 \leq s \leq t \leq b\} \\
& \longrightarrow L(\mathbb{X})
\end{aligned}
$$


where $L(\mathbb{X})$ is the space of all bounded linear operators in $\mathbb{X}$ and $\mathbb{X}$ is a Banach space. Assume that $k \in C[\Delta \times \mathbb{X}, \mathbb{X}]$ and $h \in C[I \times I \times \mathbb{X}, \mathbb{X}] . f$ is continuous. $0=t_{0}<t_{1}<$ $t_{2}<\cdots<t_{s}<t_{s+1}=b . I_{i} \in C[\mathbb{X}, \mathbb{X}],(i=1,2, \ldots, s)$ are impulsive functions, and $x\left(t_{i}^{+}\right)$and $x\left(t_{i}^{-}\right)$represent the right and the left limits of $x(t)$ at $t=t_{i}$, respectively. $B \in L[U, \mathbb{X}]$ is a bounded linear operator and the control function $u(\cdot)$ is given in $L^{2}[I, U]$ and $U$ is a Banach space. Set $T_{r}=\{x \in \mathbb{X} \mid$ $\|x\| \leq r\}$ and $B_{r}=\{x \in \mathrm{PC}[I, \mathbb{X}] \mid\|x\| \leq r\}$. $\mathrm{PC}[I, \mathbb{X}]=$ $\left\{x \in C[I, \mathbb{X}] \mid x\right.$ is continuous on $\left(t_{i}, t_{i+1}\right), i=0,1, \ldots, s$, $x\left(t_{i}^{-}\right)=x\left(t_{i}\right)$, and the right limit $x\left(t_{i}^{+}\right)$exists, $\left.i=1,2, \ldots, s\right\}$. Obviously, $\mathrm{PC}[I, \mathbb{X}]$ is a Banach space with the norm $\|x\|_{\mathrm{PC}}=$ $\sup _{t \in I}\{\|x(t)\|\}$.

Suppose that the following hypotheses are satisfied.

$\left(H_{1}\right) A(t): D(A) \rightarrow \mathbb{X}$ is a family of linear operators, generating an equicontinuous evolution system $\{G(t, s)$ : $(t, s) \in \Delta\}$; that is, $(t, s) \rightarrow\left\{G(t, s) x: x \in B_{r}\right\}$ is equicontinuous for $t>0$ and for all bounded subsets $B_{r}$.

$\left(H_{2}\right)$ For any $r>0, f$ is uniformly continuous on $I \times T_{r} \times$ $T_{r} \times T_{r}$ and $I_{i}(i=1,2, \ldots, s)$ are bounded on $T_{r}$. There exist functions $b_{p} \in C\left[I, \mathbb{R}^{+}\right](p=1,2,3,4)$ and $k^{*}$, $h^{*}>0$ such that

$$
\begin{aligned}
\|f(t, x, y, z)\| \leq & b_{1}(t)+b_{2}(t)\|x\|^{\lambda}+b_{3}(t)\|y\| \\
& +b_{4}(t)\|z\|, \\
\|k(t, s, x)\| \leq & k^{*}\|x\|^{\lambda}, \\
\|h(t, s, x)\| \leq & h^{*}\|x\|^{\lambda},
\end{aligned}
$$

where $0 \leq \lambda<1$. Define $b_{p}=\max \left\{b_{p}(t) \mid t \in I\right\}$.

$\left(H_{3}\right)$ The linear operator $W: L^{2}[I, U] \rightarrow \mathbb{X}$ is defined by

$$
W u=\int_{0}^{b} G(b, s) B u(s) d s .
$$

(i) $W$ has an invertible operator $W^{-1}$ which takes values in $L^{2}[I, U] / \mathrm{Ker} W$ and there exist positive constants $L_{B}$ and $L_{W}$ such that $\|B\| \leq L_{B}$ and $\left\|W^{-1}\right\| \leq L_{W}$;

(ii) there exists $K_{W} \in C\left[I, \mathbb{R}^{+}\right]$such that, for any bounded set $H \subset \mathbb{X}$,

$$
\alpha\left(\left(W^{-1} H\right)(t)\right) \leq K_{W}(t) \alpha(H) .
$$

Define $K_{W}=\max \left\{K_{W}(t) \mid t \in I\right\} ; \alpha(\cdot)$ represents the Kuratowski noncompactness measure.

$\left(H_{4}\right)$ There exist $l_{q} \in C\left[I, \mathbb{R}^{+}\right](q=1,2)$ such that, for any equicontinuous set, $D \subset B_{r}$, such that

$$
\begin{aligned}
& \alpha(f(t, D(s),(T D)(s),(S D)(s))) \\
& \quad \leq l_{1}(t)[\alpha(D(s))]^{\lambda}+l_{2}(t) \alpha((T D)(s)) .
\end{aligned}
$$

Define $l_{q}=\max \left\{l_{q}(t) \mid t \in I\right\}$.
Theorem 7. Assume that conditions $\left(H_{1}\right)-\left(H_{4}\right)$ hold. Then the system (25) is controllable.

Proof. Using $\left(H_{3}\right)$ (i), for every $x \in \mathrm{PC}[I, X]$, without loss of generality, define the control

$$
\begin{aligned}
& u_{0 x}(t)=W^{-1}\left[x_{1}-G(b, 0) x_{0}\right. \\
& \left.-\int_{0}^{b} G(b, s) f(s, x(s),(T x)(s),(S x)(s)) d s\right](t), \\
& u_{j x}(t)=W^{-1}\left[x_{1}-G(b, 0) x_{0}\right. \\
& -\int_{0}^{b} G(b, s) f(s, x(s),(T x)(s),(S x)(s)) d s \\
& \left.-\sum_{i=1}^{j} G\left(t, t_{i}\right) I_{i}\left(x\left(t_{i}\right)\right)\right](t),
\end{aligned}
$$

where $j=1, \ldots, s$. Define operator $Q$ as follows:

$$
\begin{aligned}
(Q x)(t)= & G(t, 0) x_{0}+\int_{0}^{t} G(t, s)\left(f+B u_{s x}\right)(s) d s \\
& +\sum_{0<t_{i}<t} G\left(t, t_{i}\right) I_{i}\left(x\left(t_{i}\right)\right) ;
\end{aligned}
$$

clearly, using the control $u_{s x}(t)$, the fixed point of operator $Q$ is a solution of the system (25), and $x_{1}=(Q x)(b)$; that is, system (25) is controllable. From the conditions we imposed, it is easy to get that operator $Q$ is continuous.

Set $\Omega_{0}=\{x \in \operatorname{PC}[I, \mathbb{X}] \mid x=\lambda Q x, 0 \leq \lambda \leq 1\}$. Assume that there exists $\lambda_{0} \in[0,1]$ such that $\bar{x}(t)=\lambda_{0}(Q \bar{x})(t)$. Next, we shall use the method of piecewise discussion.

(i) When $t \in\left[0, t_{1}\right]$,

$$
\bar{x}(t)=\lambda_{0} G(t, 0) x_{0}+\lambda_{0} \int_{0}^{t} G(t, s)\left(f+B u_{0 \bar{x}}\right)(s) d s .
$$

From Ji et al. [23], we know that there exists $L_{G}>0$ such that $\|G(t, s)\| \leq L_{G}$ for any $(t, s) \in I \times I$. Thus

$$
\begin{aligned}
\|\bar{x}(t)\| \leq & L_{G}\left\|x_{0}\right\|+L_{G} \int_{0}^{t}\left\|\left(f+B u_{0 \bar{x}}\right)(s)\right\| d s \\
\leq & L_{G}\left\|x_{0}\right\|+L_{G} \int_{0}^{t}\|f\| d s \\
& +L_{G} L_{B} \int_{0}^{t}\left\|u_{0 \bar{x}}(s)\right\| d s,
\end{aligned}
$$


where

$$
\begin{aligned}
& \int_{0}^{t}\|f\| d s \\
& \leq \int_{0}^{b} b_{1}(s) d s+b_{2} \int_{0}^{t}\|\bar{x}(s)\|^{\lambda} d s \\
& +b_{3} k^{*} t_{1} \int_{0}^{t}\|\bar{x}(s)\|^{\lambda} d s+b_{4} h^{*} t_{1} \int_{0}^{b}\|\bar{x}(s)\|^{\lambda} d s \\
& \left\|u_{0 \bar{x}}\right\|_{L^{2}} \\
& \begin{array}{l}
=\left\|W^{-1}\left[x_{1}-G(b, 0) x_{0}-\int_{0}^{b} G(b, s) f d s\right]\right\|_{L^{2}} \\
\leq L_{W}\left[\left\|x_{1}\right\|+L_{G}\left\|x_{0}\right\|+L_{G} \int_{0}^{b}\|f\| d s\right] .
\end{array}
\end{aligned}
$$

Let $u(t)=\|\bar{x}(t)\|$; then $u(t) \in C\left[\left[0, t_{1}\right], \mathbb{R}^{+}\right]$; we have

$$
\begin{aligned}
& u(t) \leq L_{G}\left\|x_{0}\right\|+L_{G} L_{B} b\left[L_{W}\left\|x_{1}\right\|+L_{W} L_{G}\left\|x_{0}\right\|\right] \\
& +L_{G}\left[\int_{0}^{b} b_{1}(s) d s+b_{2} \int_{0}^{t}[u(s)]^{\lambda} d s\right. \\
& \left.+b_{3} k^{*} t_{1} \int_{0}^{t}[u(s)]^{\lambda} d s+b_{4} h^{*} t_{1} \int_{0}^{b}[u(s)]^{\lambda} d s\right] \\
& +L_{G}^{2} L_{B} L_{W} b\left[\int_{0}^{b} b_{1}(s) d s+b_{2} \int_{0}^{b}[u(s)]^{\lambda} d s\right. \\
& \left.+b_{3} k^{*} t_{1} \int_{0}^{b}[u(s)]^{\lambda} d s+b_{4} h^{*} t_{1} \int_{0}^{b}[u(s)]^{\lambda} d s\right] .
\end{aligned}
$$

Since $\int_{1}^{\infty}\left(1 / s^{\lambda}\right) d s=\infty$, then by Theorem 5 , there exists a constant $C_{0}$ such that $u(t) \leq C_{0}, t \in\left[0, t_{1}\right]$; that is, there exists a constant $C_{0}$ independent of $u$ such that $u(t) \leq C_{0}$, $t \in\left[0, t_{1}\right]$. The above inequality implies that $\|\bar{x}(t)\| \leq C_{0}$. From $\left(H_{2}\right)$, there also exists a constant $M_{0}>0$ independent of $\bar{x}$ such that

$$
\begin{aligned}
\left\|f\left(t, \bar{x}(t), \bar{x}^{\prime}(t),(T \bar{x})(t),(S \bar{x})(t)\right)\right\| & \leq M_{0}, \\
\left\|I_{1}\left(\bar{x}\left(t_{1}\right)\right)\right\| & \leq M_{0},
\end{aligned}
$$

$\forall t \in\left[0, t_{1}\right],\|\bar{x}(t)\| \leq C_{0}$.

Thus $\left\|\bar{x}\left(t_{1}^{+}\right)\right\|=\left\|\bar{x}\left(t_{1}\right)+I_{1}\left(\bar{x}\left(t_{1}\right)\right)\right\| \leq C_{0}+M_{0}$.

(ii) When $t \in\left(t_{1}, t_{2}\right]$,

$$
\bar{v}(t)= \begin{cases}\bar{x}(t), & t \in\left(t_{1}, t_{2}\right], \\ \bar{x}\left(t_{1}^{+}\right), & t=t_{1} .\end{cases}
$$

Then $\bar{v}(t) \in C\left[\left[t_{1}, t_{2}\right], \mathbb{X}\right]$ and

$$
\begin{aligned}
\bar{v}(t)= & \lambda_{0} G(t, 0) x_{0}+\lambda_{0} \int_{0}^{t_{1}} G(t, s)\left(f+B u_{1 \bar{v}}\right)(s) d s \\
& +\lambda_{0} \int_{t_{1}}^{t} G(t, s)\left(f+B u_{1 \bar{v}}\right)(s) d s \\
& +\lambda_{0} G\left(t, t_{1}\right) I_{1}\left(x\left(t_{1}\right)\right) .
\end{aligned}
$$

From results (38) and similar to the proof of (i), we can know that there exists $C_{1}>0$ that does not depend on $\bar{x}$ such that $\|\bar{v}(t)\| \leq C_{1}, t \in\left[t_{1}, t_{2}\right]$. So $\|\bar{x}(t)\| \leq C_{1}, t \in\left(t_{1}, t_{2}\right]$.

By the same method as above, we can prove that there exists a constant $C_{s}>0$ that does not depend on $\bar{x}$ such that

$$
\|\bar{x}(t)\| \leq C_{s}, \quad t \in\left(t_{s}, b\right] .
$$

Let $C=\max \left\{C_{i} \mid 0 \leq i \leq s\right\}$; then $\|\bar{x}(t)\| \leq C, t \in I$. Thus $\Omega_{0}$ is a bounded set in PC $[I, \mathbb{X}]$. Take $R>C$; let $\Omega=\{x \in$ $\left.\mathrm{PC}[I, \mathbb{X}] \mid\|x\|_{\mathrm{PC}}<R\right\}$; obviously $\Omega$ is a bounded open set in $\operatorname{PC}[I, \mathbb{X}]$ and $\theta \in \Omega$. From the choice of $R$, we know that if $x \in \partial \Omega$ and $\lambda \in[0,1]$, we have $x \neq \lambda Q x$.

Let $H \subset \bar{\Omega}$ be a countable set and $H \subset \overline{c o}(\{\theta\} \bigcup Q(H))$. By $\left(H_{1}\right)$ and $\left(H_{2}\right)$, it is easy to see that $Q(H)$ is equicontinuous on each $\left[t_{i}, t_{i+1}\right],(i=0,1,2, \ldots, s)$.

Next, we shall prove that $(Q H)(t)$ is relatively compact for each $\left[t_{i}, t_{i+1}\right]$. In the same way, we discuss step by step as follows.

(i) When $t \in\left[t_{0}, t_{1}\right]$

$$
\begin{aligned}
& \alpha(H(t)) \leq \alpha((Q H)(t)) \leq \alpha\left(G(t, 0) x_{0} \mid x \in H\right) \\
& +\alpha\left(\int_{0}^{t} G(t, s)\left(f+B u_{0 x}\right)(s) d s \mid x \in H\right) \\
& \quad \leq 2 L_{G} l_{1} \int_{0}^{t}[\alpha(H(s))]^{\lambda} d s \\
& +2 L_{G} l_{2} k^{*} b \int_{0}^{t} \alpha(H(s)) d s \\
& \quad+L_{G} L_{B} \int_{0}^{t} \alpha\left(u_{0 x} \mid x \in H\right) d s, \\
& \alpha\left(u_{0 x} \mid x \in H\right) \leq K_{W}\left(2 L_{G} l_{1} \int_{0}^{b}[\alpha(H(s))]^{\lambda} d s\right. \\
& \left.\quad+2 L_{G} l_{2} k^{*} b \int_{0}^{t} \alpha(H(s)) d s\right) .
\end{aligned}
$$

Let $m(t)=\alpha(H(t)), t \in\left[0, t_{1}\right]$; then $m(t) \in C\left[\left[0, t_{1}\right], \mathbb{R}^{+}\right]$. Thus

$$
\begin{aligned}
m(t) \leq & 2 L_{G} l_{1} \int_{0}^{t}[\alpha(H(s))]^{\lambda} d s \\
& +2 L_{G} l_{2} k^{*} b \int_{0}^{t} \alpha(H(s)) d s
\end{aligned}
$$




$$
\begin{aligned}
& +2 K_{W} L_{G} l_{1} b \int_{0}^{t}[\alpha(H(s))]^{\lambda} d s \\
& +2 K_{W} L_{G} l_{2} b k^{*} t \int_{0}^{t} \alpha(H(s)) d s .
\end{aligned}
$$

By Remark $6(\mathrm{i})$, we have $m(t) \equiv 0, t \in\left[0, t_{1}\right]$. Thus $\alpha(H(t))=$ 0 ; that is, $H$ is a relatively compact set in $\mathrm{PC}\left[\left[0, t_{1}\right], \mathbb{X}\right]$. Since $I_{1} \in C[\mathbb{X}, \mathbb{X}], \alpha\left(I_{1}\left(H\left(t_{1}\right)\right)\right)=0$, then $H\left(t_{1}\right)$ is a relatively compact set in $\mathbb{X}$.

(ii) For $t \in\left(t_{1}, t_{2}\right]$, we know

$$
\begin{aligned}
\alpha(H(t)) & \\
\leq & \alpha\left(G(t, 0) x_{0} \mid x \in H\right) \\
& +\alpha\left(\int_{0}^{t} G(t, s)\left(f+B u_{1 x}\right)(s) d s \mid x \in H\right) \\
& +\alpha\left(I_{1}\left(x\left(t_{1}\right)\right) \mid x \in H\right) \\
\leq & 2 L_{G} l_{1} \int_{0}^{t}[\alpha(H(s))]^{\lambda} d s \\
& +2 L_{G} l_{2} k^{*} b \int_{0}^{t} \alpha(H(s)) d s \\
& +L_{G} L_{B} \int_{0}^{t} \alpha\left(u_{1 x} \mid x \in H\right) d s .
\end{aligned}
$$

Similar to the proof of (i), we can deduce that $m(t) \equiv 0$, $t \in\left[t_{1}, t_{2}\right]$. Therefore $\alpha(H(t))=0, t \in\left[t_{1}, t_{2}\right]$. In particular, $\alpha\left(H\left(t_{2}\right)\right)=0$, so $H$ is a relatively compact set in $C\left[\left[t_{1}, t_{2}\right], \mathbb{X}\right]$. Similarly, we can show that $H$ is a relatively compact set in $C\left[\left[t_{i}, t_{i+1}\right], \mathbb{X}\right](i=2,3, \ldots, s)$. Thus $H$ is a relatively compact set in $\mathrm{PC}[I, \mathbb{X}]$.

In conclusion, we deduce that $Q$ has at least one fixed point in $\Omega$ by the Mönch fixed point theorem; that is, system (25) has at least one mild solution in $\mathrm{PC}[I, \mathbb{X}]$. Thus system (25) is controllable on $I$.

\section{The Uniqueness and Global Existence of Solutions}

Consider the following integral equation:

$$
\begin{gathered}
u(t)=k(t)+\int_{0}^{\alpha(t)} f(s) w(u(s)) d s \\
+\int_{0}^{\beta(t)} g(s) w(u(s)) d s
\end{gathered}
$$

where $k, f, g \in C\left(\mathbb{R}^{+}, \mathbb{R}^{+}\right)$with $w(0)=0$ and $\alpha, \beta \in$ $C^{1}\left(\mathbb{R}^{+}, \mathbb{R}^{+}\right)$are nondecreasing with $\alpha(t), \beta(t) \leq t$ on $\mathbb{R} \mathbb{R}^{+}$. Assume that (45) has a solution $u \in C\left([0, T], \mathbb{R}^{+}\right)$on some maximal interval of existence $[0, T]$. Moreover, if $T<\infty$,

$$
\limsup _{t \rightarrow T} u(t)=\infty \text {. }
$$

Theorem 8. Assume that

$$
\|w(x)-w(y)\| \leq Z(\|x-y\|)
$$

with $Z \in C\left(\mathbb{R}^{+}, \mathbb{R}^{+}\right)$nondecreasing, $Z(x)>0$ for $x>0$. If

$$
\int_{0}^{1} \frac{1}{Z(s)} d s=\int_{1}^{\infty} \frac{1}{Z(s)} d s=\infty,
$$

then (45) has a unique solution defined on $\mathbb{R}^{+}$. Moreover, if $k$ is bounded on $\mathbb{R}^{+}$and if either $\alpha, \beta$ is bounded on $\mathbb{R}^{+}$or $\int_{0}^{\infty} f(s) d s, \int_{0}^{\infty} g(s) d s<\infty$, then its solution is bounded on $\mathbb{R}^{+}$.

Proof. Suppose that, on some interval $\left[0, t_{0}\right],(45)$ has two solutions $u_{1}, u_{2} \in C\left(\left[0, t_{0}\right], \mathbb{R}^{+}\right)$; we obtain

$$
\begin{aligned}
& u_{1}(t)-u_{2}(t) \\
& =\int_{0}^{\alpha(t)} f(s)\left[w\left(u_{1}(s)\right)-w\left(u_{2}(s)\right)\right] d s \\
& \quad+\int_{0}^{\beta(t)} g(s)\left[w\left(u_{1}(s)\right)-w\left(u_{2}(s)\right)\right] d s .
\end{aligned}
$$

Denote $u(t)=\left\|u_{1}(t)-u_{2}(t)\right\|$; we have

$$
\begin{aligned}
u(t) \leq & \int_{0}^{\alpha(t)} f(s) Z(u(s)) d s \\
& +\int_{0}^{\beta(t)} g(s) Z(u(s)) d s .
\end{aligned}
$$

Set

$$
G(r)=\int_{1}^{r} \frac{1}{Z(s)} d s, \quad r>0 .
$$

Then $G(0)=-\infty$ and $G(\infty)=\infty$. There exists $\epsilon>0$, where

$$
\begin{aligned}
u(t) \leq & \epsilon+\int_{0}^{\alpha(t)} f(s) Z(u(s)) d s \\
& +\int_{0}^{\beta(t)} g(s) Z(u(s)) d s, \quad 0 \leq t \leq t_{0} .
\end{aligned}
$$

From Theorem 5, we know that

$$
\begin{gathered}
u(t) \leq G^{-}\left(G(\epsilon)+\int_{0}^{\alpha(t)} f(s) Z(u(s)) d s\right. \\
\left.+\int_{0}^{\beta(t)} g(s) Z(u(s)) d s\right), \quad 0 \leq t \leq t_{0} .
\end{gathered}
$$

From Remark 6, $u(t) \rightarrow 0, \epsilon \rightarrow 0$; then $u_{1}(t)=u_{2}(t)$, and the uniqueness of the solution can be obtained.

Next, we will show that the solution is global; that is, $T=$ $\infty$, where $T$ is the maximal time of existence. If $T<\infty$, we set $k_{0}=\max _{0 \leq t \leq T}\{k(t)\}$, and we obtain that

$$
\begin{aligned}
u(t) \leq & k_{0}+\int_{0}^{\alpha(t)} f(s) Z(u(s)) d s \\
& +\int_{0}^{\beta(t)} g(s) Z(u(s)) d s, \quad 0 \leq t<T,
\end{aligned}
$$


as $w(u(s))=w(u(s))-w(0) \leq Z(u(s))$ for $0 \leq t<T$. By Lemma 1 and $u \in C\left[[0, T], \mathbb{R}^{+}\right]$, we deduce that

$$
\begin{array}{r}
u(t) \leq G^{-}\left(G\left(k_{0}\right)+\int_{0}^{\alpha(t)} f(s) d s+\int_{0}^{\beta(t)} g(s) d s\right), \\
0 \leq t<T .
\end{array}
$$

Since $k$ is bounded on $\mathbb{R}^{+}$and either $\alpha, \beta$ is bounded on $\mathbb{R}^{+}$or $\int_{0}^{\infty} f(s) d s, \int_{0}^{\infty} g(s) d s<\infty$ satisfies; then $u(t)$ is bounded on $\mathbb{R}^{+}$. Thus the previous inequality (55) contradicts (46). Thus the global existence is proved.

\section{Conclusions}

This paper generalizes a more general Gronwall-like inequality with a Fredholm operator. Using the proposed inequality, we solve a difficult problem in the research of the controllability of integrodifferential systems of mixed type in Banach space. Meanwhile, we also prove the uniqueness and global existence of solutions for a class of integral equations. Therefore, the results we obtained are very important and powerful tools. However, it should be more useful than we can imagine in qualitative properties of many other nonlinear problems, such as existence, estimation of solutions, dependence of solutions on parameters in nonlinear analysis, and control.

\section{Conflict of Interests}

The authors declare that there is no conflict of interests regarding the publication of this paper.

\section{References}

[1] B. G. Pachpatte, Inequalities for Differential and Integral Equations, Academic Press, New York, NY, USA, 1998.

[2] T. H. Gronwall, "Note on the derivatives with respect to a parameter of the solutions of a system of differential equations," The Annals of Mathematics. Second Series, vol. 20, no. 4, pp. 292296, 1919.

[3] R. Bellman, "The stability of solutions of linear differential equations," Duke Mathematical Journal, vol. 10, pp. 643-647, 1943.

[4] Y. Jalilian and R. Jalilian, "Existence of solution for delay fractional differential equations," Mediterranean Journal of Mathematics, vol. 10, no. 4, pp. 1731-1747, 2013.

[5] O. Lipovan, "Integral inequalities for retarded Volterra equations," Journal of Mathematical Analysis and Applications, vol. 322, no. 1, pp. 349-358, 2006.

[6] W.-S. Wang, "A generalized retarded Gronwall-like inequality in two variables and applications to BVP," Applied Mathematics and Computation, vol. 191, no. 1, pp. 144-154, 2007.

[7] R. P. Agarwal, S. Deng, and W. Zhang, "Generalization of a retarded Gronwall-like inequality and its applications," Applied Mathematics and Computation, vol. 165, no. 3, pp. 599-612, 2005.

[8] S. Guo, I. Moroz, L. Si, and L. Han, "Several integral inequalities and their applications in nonlinear differential systems," Applied Mathematics and Computation, vol. 219, no. 9, pp. 4266-4277, 2013.
[9] Y.-H. Lan and Y. Zhou, "High-order $\mathscr{D}^{\alpha}$-type iterative learning control for fractional-order nonlinear time-delay systems," Journal of Optimization Theory and Applications, vol. 156, no. 1, pp. 153-166, 2013.

[10] Q. Feng and F. Meng, "Some new Gronwall-type inequalities arising in the research of fractional differential equations," Journal of Inequalities and Applications, vol. 2013, article 429, 2013.

[11] K. Zheng, W. Feng, and C. Guo, "Some new nonlinear weakly singular inequalities and applications to Volterra-type difference equation," Abstract and Applied Analysis, vol. 2013, Article ID 912874, 6 pages, 2013.

[12] B. Zheng, "Some new Gronwall-Bellman-type inequalities based on the modified Riemann-Liouville fractional derivative," Journal of Applied Mathematics, vol. 2013, Article ID 341706, 8 pages, 2013.

[13] H. Wang and B. Zheng, "Some new dynamic inequalities and their applications in the qualitative analysis of dynamic equations," WSEAS Transactions on Mathematics, vol. 12, no. 10, pp. 967-978, 2013.

[14] Y.-H. Kim, "Gronwall, Bellman and Pachpatte type integral inequalities with applications," Nonlinear Analysis: Theory, Methods \& Applications, vol. 71, no. 12, pp. e2641-e2656, 2009.

[15] H. Ye and J. Gao, "Henry-Gronwall type retarded integral inequalities and their applications to fractional differential equations with delay," Applied Mathematics and Computation, vol. 218, no. 8, pp. 4152-4160, 2011.

[16] J. Wang, X. Xiang, W. Wei, and Q. Chen, “The generalized Gronwall inequality and its application to periodic solutions of integrodifferential impulsive periodic system on Banach space," Journal of Inequalities and Applications, vol. 2008, Article ID 430521, 22 pages, 2008.

[17] Y. Peng, X. Xiang, and W. Wei, "Nonlinear impulsive integrodifferential equations of mixed type with time-varying generating operators and optimal controls," Dynamic Systems and Applications, vol. 16, no. 3, pp. 481-496, 2007.

[18] O. Lipovan, "A retarded Gronwall-like inequality and its applications," Journal of Mathematical Analysis and Applications, vol. 252, no. 1, pp. 389-401, 2000.

[19] H. Ye, J. Gao, and Y. Ding, "A generalized Gronwall inequality and its application to a fractional differential equation," Journal of Mathematical Analysis and Applications, vol. 328, no. 2, pp. 1075-1081, 2007.

[20] L. Liu, "Iterative method for solutions and coupled quasisolutions of nonlinear integro-differential equations of mixed type in Banach spaces," Nonlinear Analysis: Theory, Methods \& Applications, vol. 42, pp. 583-598, 2000.

[21] H. Qin and X. Zuo, "Controllability of nonlocal boundary conditions for impulsive differential systems of mixed type in banach spaces," in Proceedings of the 10th IEEE International Conference on Control and Automation (ICCA '13), pp. 10151019, IEEE, Hangzhou, China, June 2013.

[22] Y. Li and H. Zhang, "The solutions of initial value problems for nonlinear second order integro-differential equations of mixed type in Banach spaces," Journal of Shandong University (Natural Science), vol. 45, pp. 93-98, 2010 (Chinese).

[23] S. Ji, G. Li, and M. Wang, "Controllability of impulsive differential systems with nonlocal conditions," Applied Mathematics and Computation, vol. 217, no. 16, pp. 6981-6989, 2011.

[24] L. Liu, F. Guo, C. Wu, and Y. Wu, "Existence theorems of global solutions for non-linear Volterra type integral equations 
in Banach spaces," Journal of Mathematical Analysis and Applications, vol. 309, no. 2, pp. 638-649, 2005.

[25] F. Guo, L. Liu, Y. Wu, and P.-F. Siew, "Global solutions of initial value problems for nonlinear second-order impulsive integro-differential equations of mixed type in Banach spaces," Nonlinear Analysis. Theory, Methods \& Applications, vol. 61, no. 8, pp. 1363-1382, 2005.

[26] L. Liu, C. Wu, and F. Guo, "Existence theorems of global solutions of initial value problems for nonlinear integrodifferential equations of mixed type in banach spaces and applications," Computers \& Mathematics with Applications, vol. 47, no. 1, pp. 13-22, 2004. 


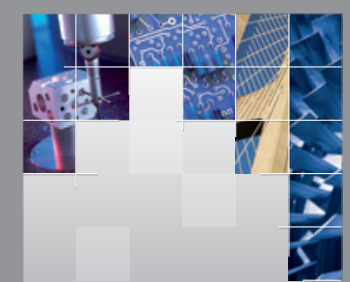

\section{Enfincering}
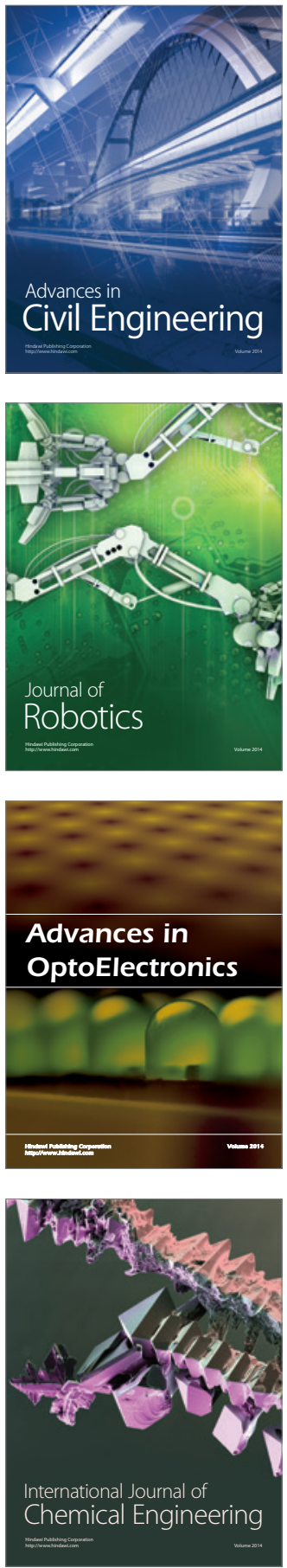

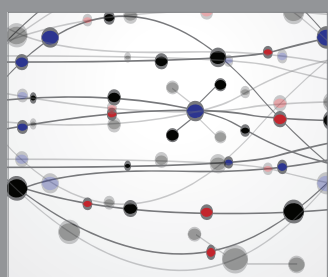

The Scientific World Journal

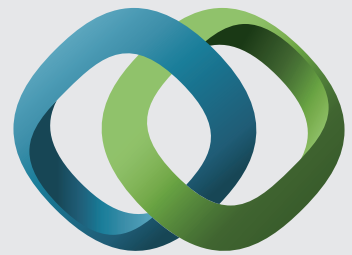

\section{Hindawi}

Submit your manuscripts at

http://www.hindawi.com
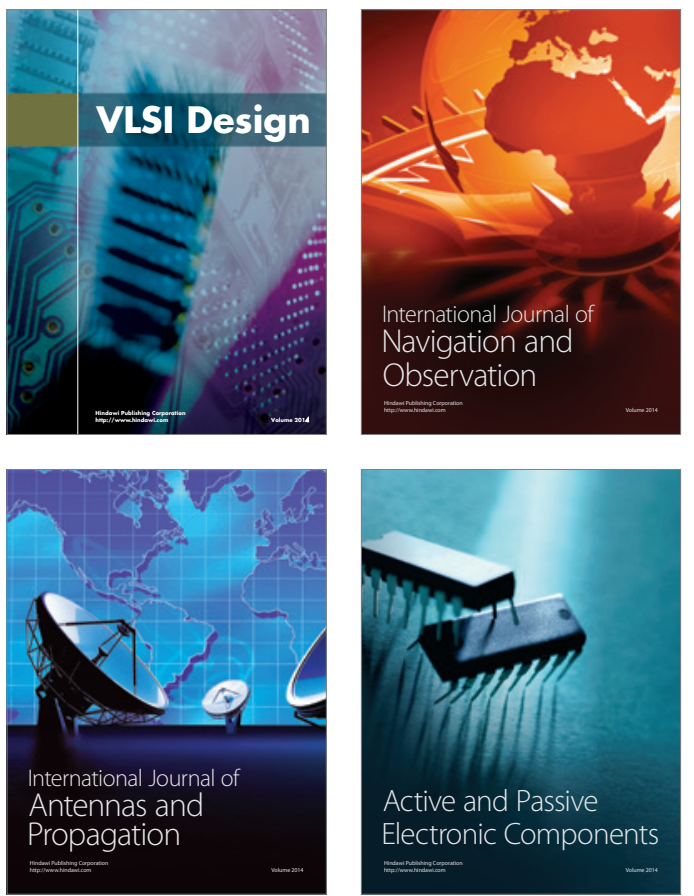
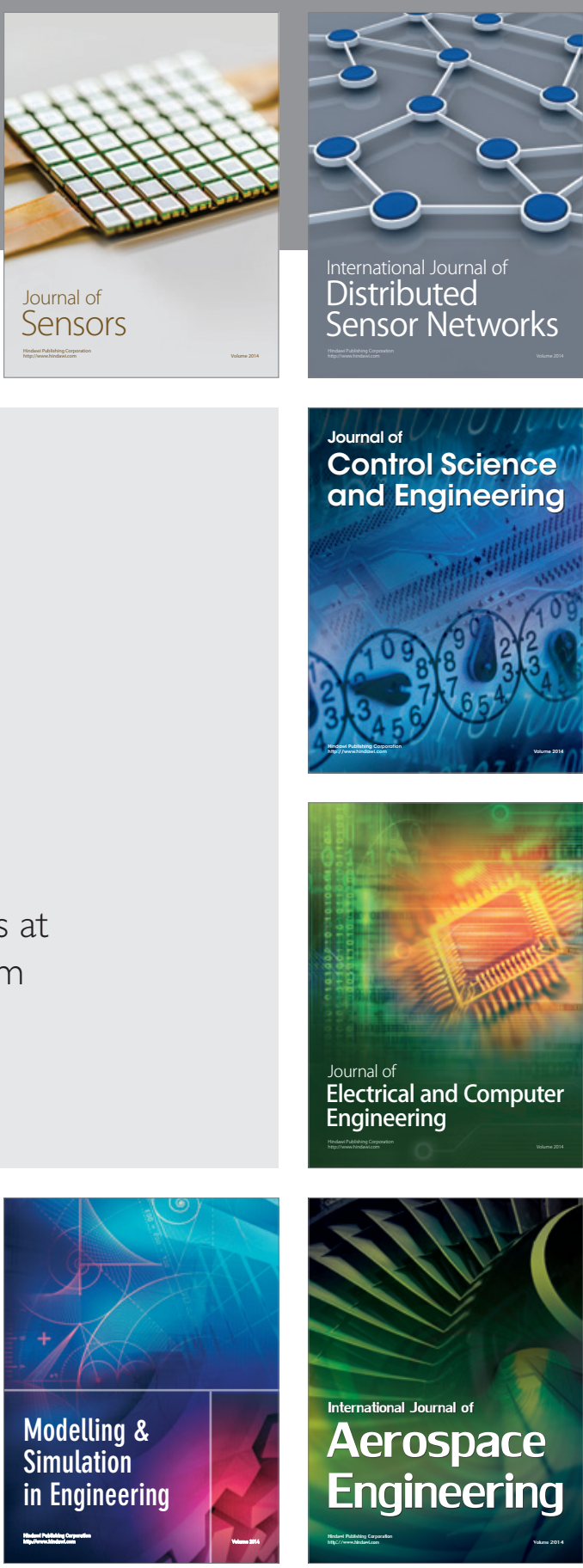

International Journal of

Distributed

Sensor Networks

Journal of

Control Science

and Engineering
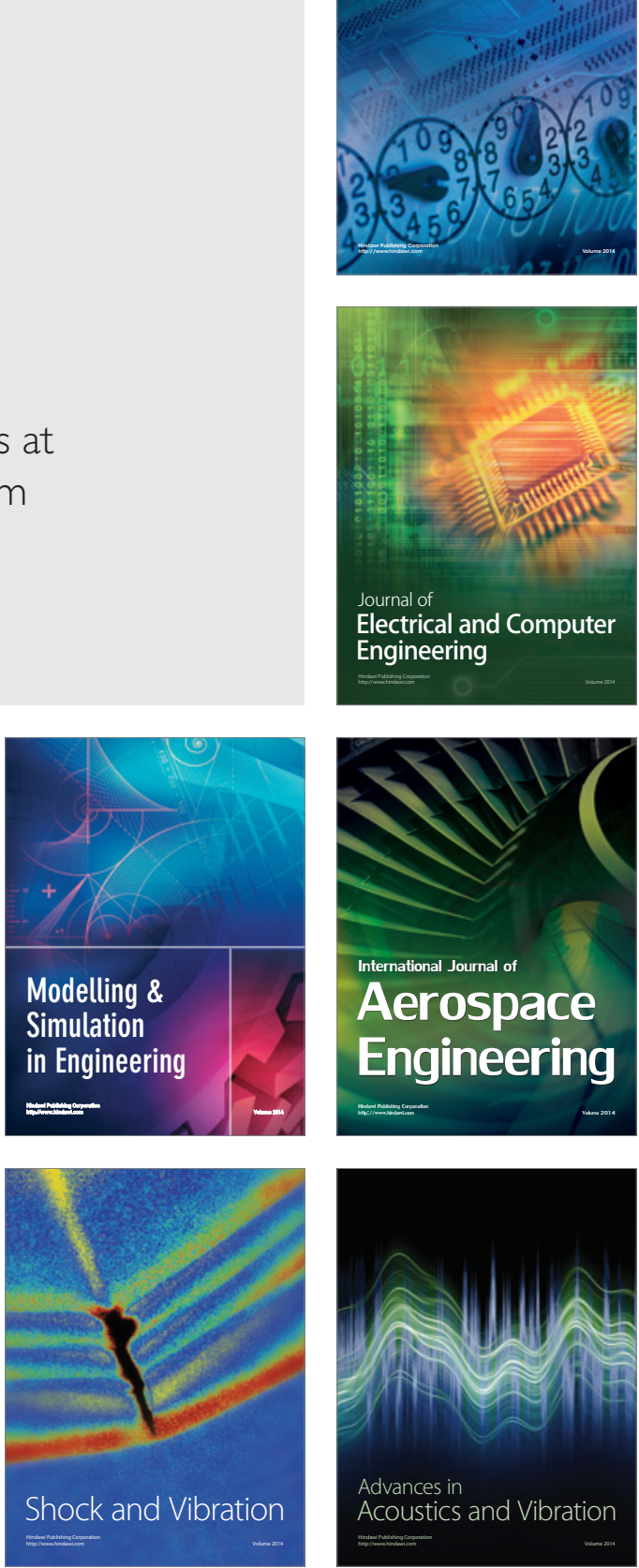